\title{
Ultrasonic Welding
}

\author{
FRANK BALLE ${ }^{1,2}$ \\ 1.--Institute of Materials Science and Engineering, University of Kaiserslautern, Kaiserslautern, \\ Germany. 2.—e-mail: balle@mv.uni-kl.de
}

This issue of JOM presents selected papers on the use of ultrasonic metal welding for solid-state joining of metals to metals as well as dissimilar materials. In contrast to the well-known and established ultrasonic welding for polymers, the direction of the ultrasonic oscillation is not perpendicular but parallel to the surface of the materials to be welded in case of ultrasonic metal welding. This pressure welding technology was invented and patented in the early 1930 s. It is a solid-state welding technique, where the formation of the bond occurs as a result of a moderate static pressure and a superimposed ultrasonic oscillation without reaching their melting points. In comparison with fusion welding or even other joining techniques such as adhesive bonding or brazing, ultrasonic welding is characterized by low energy input, consequently low temperatures in the welding zone, and very short welding times as well as moderate investment costs. The high-frequency relative motion between the parts forms a solid-state weld through progressive shearing and high plastic deformation between surface asperities that disperses oxides and contaminants. Consequently, an increasing area of pure metal contact and bonding of the adjacent surfaces will be realized. The process is excellent for joining foils, thin sheets to thicker sheets with spot or seam welds, as well as wires to connectors in circuit boards or for cable harnesses.

Most metals and their alloys can be ultrasonically welded. Furthermore, dissimilar metal combinations are readily welded with high-power ultrasonics. Regarding efficiency, automation capability, and achievable mechanical and technological properties, ultrasonic metal welding is a very attractive alternative to existing joining techniques for multi-material systems. It has now been used for selected industrial applications in the electrical industry, the automotive and aircraft sectors, and engineering in general.

The following four papers cover the latest advances on joining wires and especially dissimilar materials.

The first paper, "Ultrasonic Welding of Hybrid Joints" by Wagner and colleagues, gives an overview of all available modern ultrasonic welding systems and the most important characteristics of joint quality. Besides the ultrasonic welding of dissimilar metals such as $\mathrm{Al}$ to $\mathrm{Cu}$ or $\mathrm{Al}$ to steels, welds between newly developed materials such as aluminum foam sandwiches (AFS) or flat flexible cables (FFC) are described. Furthermore, the mechanical properties of ultrasonically welded joints of glass and even ceramics to different sheet metals are discussed. Finally, selected results about the occurring bonding mechanisms are presented.

"Effect of Zinc Coatings on Joint Properties and Interface Reactions in Aluminum to Steel Ultrasonic Spot Welding" by Haddadi et al. from University of Manchester (UK) addresses the weldability of different zinc-coated steels with aluminum alloys. A strong influence of the type of zinc coating is observed and investigated in detail. The achievable strength for different ultrasonically welded Al to steel joints is presented as well as additional microstructural analysis of the interfacial reactions.

In the third paper, also from University of Manchester, "Optimization of Aluminum to Magnesium Ultrasonic Spot Welding" by Panteli and colleagues, the effect of process variables and energy input on joint formation between $\mathrm{Al}$ alloy AA6111 and $\mathrm{Mg}$ alloy AZ31 is introduced. Besides determining suitable process parameters, interface reactions and the formation of a significant intermetallic reaction layer are investigated by scanning electron microscopy (SEM), energy dispersive spectroscopy (EDS) as well as x-ray diffraction (XRD) analysis.

Finally, in the work of Heinz et al., "Ultrasonic Welding of Wires and Cables," recent advances on ultrasonic spot welding of $\mathrm{Al}$ wires with large crosssections up to $80 \mathrm{~mm}^{2}$ are presented. A major challenge is the reduction of unintentional adherence between the welding tool and $\mathrm{Al}$ wires by developing multilayer coatings of the sonotrode tip. The second part of the article reports on ultrasonically welded FFC by using ultrasonic spot and torsion welding systems. A central purpose of the work is the development of a two-stage process which enables welding through the insulation of the FFC without weakening the base material. 